\title{
LARGE DEVIATIONS FOR THE GRAPH DISTANCE IN SUPERCRITICAL CONTINUUM PERCOLATION
}

\author{
CHANG-LONG YAO, ${ }^{* * *}$ \\ GE CHEN * AND \\ TIAN-DE GUO, * Graduate University of Chinese Academy of Sciences
}

\begin{abstract}
Denote the Palm measure of a homogeneous Poisson process $\mathscr{H}_{\lambda}$ with two points 0 and $x$ by $\mathrm{P}_{0, x}$. We prove that there exists a constant $\mu \geq 1$ such that $\mathrm{P}_{0, x}\left(D(0, x) / \mu\|x\|_{2} \notin\right.$ $\left.(1-\varepsilon, 1+\varepsilon) \mid 0, x \in C_{\infty}\right)$ exponentially decreases when $\|x\|_{2}$ tends to $\infty$, where $D(0, x)$ is the graph distance between 0 and $x$ in the infinite component $C_{\infty}$ of the random geometric graph $G\left(\mathscr{H}_{\lambda} ; 1\right)$. We derive a large deviation inequality for an asymptotic shape result. Our results have applications in many fields and especially in wireless sensor networks.
\end{abstract}

Keywords: Continuum percolation; chemical distance; shape theorem; large deviation inequality

2010 Mathematics Subject Classification: Primary 60K35

Secondary 82B43

\section{Introduction}

Standard first passage percolation (FPP) was formulated by Hammersley and Welsh [9] as a simplified model for the spread of a fluid in a porous medium. Then some new FPP models in a random environment were studied. Based on homogeneous Poisson point processes, VahidiAsl and Wierman [16] introduced a class of FPP models for the Poisson-Voronoi tessellations, Howard and Newman [10], [11] established a Euclidean FPP, and Baccelli and Bordenave [2] analyzed a class of spatial random spanning trees built on the Poisson point processes of the plane. The authors proved shape theorems for these continuum FPP models. Garet and Marchand [4]-[6] introduced a discrete FPP model based on an infinite Bernoulli percolation cluster. They obtained a large deviation theorem for the chemical distance between two points in that cluster and derived a large deviation inequality for the corresponding asymptotic shape result.

In this paper we present another new FPP model based on the infinite component in the continuum percolation. Analogous to the models above, it is natural to obtain a shape theorem for our model. The large deviations of the graph distance and the shape theorem are important properties of the infinite component and can be applied to communication networks, particularly to the large-scale randomly distributed wireless sensor networks (WSNs), which can be modeled using the infinite component of continuum percolation. For instance, Dousse [3] constructed a two-dimensional continuum percolation to study a sort of WSN and obtained some results about the delay of the networks. In our model, we think of the Poisson points as the locations

Received 30 April 2010; revision received 20 October 2010.

* Postal address: School of Mathematical Science, Graduate University of Chinese Academy of Sciences, 100049,

Beijing, P. R. China.

** Email address: deducemath@126.com 
of the sensor nodes in the WSNs: every node has the same transmitting radius and can transmit data to the nodes in their transmitting range directly. The messages from the nodes will be transmitted to an appointed cluster node by a multihop path in the network. Here we suppose that the appointed cluster node belongs to the infinite component. As a result, the other finite components will be omitted because the nodes in these components cannot transmit messages to the cluster node. For applications to WSNs, our model is more practical than the model of the radial spanning trees of Poisson point processes proposed by Baccelli and Bordenave [2], since they did not consider the transmitting radius restriction.

In a future paper we will provide the moderate deviation for the graph distance and analyze the asymptotic direction of the shortest path.

\section{Definitions and main results}

Now let us introduce some usual notation and the percolation models.

Throughout this paper, we assume that $\|\cdot\|$ is the $l_{2}$-norm and that the dimension, $d$, is greater than or equal to 2 . Suppose that $A$ is a Borel set in $\mathbb{R}^{d}$. We use $|A|$ to denote its cardinality if $A$ is a finite point set; otherwise we use $|A|$ to denote its Lebesgue measure.

For $x \in \mathbb{R}^{d}$ and $r>0$, we define a ball in $\mathbb{R}^{d}$ by

$$
B(x, r):=\left\{y \in \mathbb{R}^{d}:\|x-y\| \leq r\right\} .
$$

A path in the graph $G(V, E)$ is a sequence of vertices $\ell:=\left(x_{0}, x_{1}, \ldots, x_{n}\right)$ such that $\left\{x_{i-1}, x_{i}\right\}$ lies in $E$ for each $i=1,2, \ldots, n$. The length of $\ell$ is the number, $n$, of edges and is denoted by $|\ell|$. In this paper, the terminology cluster is identical to component in graph theory. If two vertices $x$ and $y$ are in the same component, we write $x \leftrightarrow y$.

Let us recall the Bernoulli percolation model. For each pair $\{x, y\}$ of points in $\mathbb{Z}^{d}$ with $\|x-y\|_{1}=1$, we add an undirected edge between them. Define a $d$-dimensional cubic lattice $\mathbb{L}^{d}:=\left(\mathbb{Z}^{d}, \mathbb{E}^{d}\right)$, where $\mathbb{E}^{d}$ stands for the set of all edges. If $\|x-y\|_{1}=1$, we say that $x$ and $y$ are adjacent, and write $x \sim y$. If all edges (vertices) of $\mathbb{L}^{d}$ are open with probability $p$ and closed with probability $1-p$ independently of each other, we call the model Bernoulli bond (site) percolation. The corresponding probability measure on $\{0,1\} \mathbb{E}^{\mathbb{E}^{d}}$ is denoted by $\mathrm{P}_{p}$.

Now we introduce the continuum percolation model (see [14, pp. 188-190]). Given a point set $\mathcal{X} \subset \mathbb{R}^{d}$, we denote by $G(\mathcal{X} ; 1)$ the undirected graph with vertex set $\mathcal{X}$ and undirected edges connecting all point pairs $\{x, y\}$ such that $\|x-y\| \leq 1$. The metric diameter of $G(X ; 1)$ is $\sup \{\|x-y\|: x, y \in G\}$. Let $\mathscr{H}_{\lambda}$ denote a homogeneous Poisson process of intensity $\lambda$ on $\mathbb{R}^{d}$. Then we can construct an infinite random geometric graph $G\left(\mathscr{H}_{\lambda} ; 1\right)$. For $s>0$, define $B(s)$ to be the box of side $s$ centered at the origin, and let $\mathscr{H}_{\lambda, s}$ be the restriction of $\mathscr{H}_{\lambda}$ to the box $B(s)$. In other words, define

$$
B(s):=\left[-\frac{1}{2} s, \frac{1}{2} s\right]^{d}, \quad \mathscr{H}_{\lambda, s}:=\mathscr{H}_{\lambda} \cap B(s) .
$$

Let $\mathscr{H}_{\lambda, 0}$ denote the point process $\mathscr{H}_{\lambda} \cup\{0\}$, where 0 is the origin in $\mathbb{R}^{d}$. For $k \in \mathbb{N}$, let $p_{k}(\lambda)$ denote the probability that the component of $G\left(\mathscr{H}_{\lambda, 0} ; 1\right)$ containing the origin is of order $k$. The percolation probability, $p_{\infty}(\lambda)$, is the probability that 0 lies in an infinite component of the graph $G\left(\mathscr{H}_{\lambda, 0} ; 1\right)$, and is defined by

$$
p_{\infty}(\lambda)=1-\sum_{k=1}^{\infty} p_{k}(\lambda) .
$$


The critical intensity, $\lambda_{c}$, is defined by

$$
\lambda_{\mathrm{c}}=\inf \left\{\lambda>0: p_{\infty}(\lambda)>0\right\} .
$$

The fundamental result of continuum percolation says that $0<\lambda_{\mathrm{c}}<\infty$, provided that $d \geq 2$. If $\lambda>\lambda_{\mathrm{c}}$ almost surely, there is a unique infinite component in continuum percolation, which we denote by $C_{\infty}$. In some cases, we may add several points $x_{1}, \ldots, x_{n} \in \mathbb{R}^{d}$ into $\mathscr{H}_{\lambda}$; for notational simplicity, we also use $C_{\infty}$ to denote the infinite component of $G\left(\mathscr{H}_{\lambda} \cup\right.$ $\left.\left\{x_{1}, \ldots, x_{n}\right\} ; 1\right)$.

The theorem of Slyvniak states that the Palm distribution $\mathrm{P}_{x}$ of a Poisson process $\mathscr{H}_{\lambda}$ of distribution $\mathrm{P}$ is given by

$$
\mathrm{P}_{x}=\mathrm{P} * \delta_{x} \quad \text { for } x \in \mathbb{R}^{d} .
$$

Here ' $*$ ' denotes the convolution of distributions, which corresponds to the superposition of point processes. The $\delta_{x}$ term denotes the distribution of the degenerate point process that consists solely of the (nonrandom) point $x$. The $n$-fold Palm distribution $\mathrm{P}_{x_{1}, \ldots, x_{n}}$ of a Poisson process with distribution $\mathrm{P}$ is, analogously to (2.1), equal to

$$
\mathrm{P} * \delta_{x_{1}} * \cdots * \delta_{x_{n}} \quad \text { for } x_{1}, \ldots, x_{n} \in \mathbb{R}^{d}
$$

see [15, p. 124]. For all $x, y \in G\left(\mathscr{H}_{\lambda} ; 1\right)$, we introduce the graph distance between $x$ and $y$ as

$$
D(x, y):=\inf \left\{|\ell|: x, y \in \ell, \ell \text { is a path in } G\left(\mathscr{H}_{\lambda} ; 1\right)\right\} .
$$

Note that the graph distance corresponds to the chemical distance in the Bernoulli percolation introduced in [1] and [5]. It is natural to think of the graph distance as a special travel time in an FPP model.

Our first result concerns the asymptotic behavior of the graph distance between two points in the infinite component of continuum percolation. Analogously to the classic FPP, we obtain the following large deviation result.

Theorem 2.1. Suppose that $\lambda>\lambda_{\mathrm{c}}$ and that $\mu$ is the constant given in Lemma 3.5 below. Then, for all $\varepsilon>0$,

$$
\limsup _{\|x\| \rightarrow \infty} \frac{\log \mathrm{P}_{0, x}(0 \leftrightarrow x, D(0, x) / \mu\|x\| \notin(1-\varepsilon, 1+\varepsilon))}{\|x\|}<0 .
$$

Our second result concerns the asymptotic shape of the points which can be reached by $k$ hops from a given point of the infinite component.

Theorem 2.2. (Shape theorem.) Suppose that $\lambda>\lambda_{\mathrm{c}}$ and that $\mu$ is the constant given in Lemma 3.5 below. Let $C_{k}:=G\left(\left\{x \in C_{\infty}: D(0, x) \leq k\right\} ; 1\right)$. Then, given any $\varepsilon>0$, there exists a constant $c>0$ such that

$$
\begin{aligned}
& \mathrm{P}_{0}\left(\left(C_{\infty} \cap B\left(0, \frac{(1-\varepsilon) k}{\mu}\right)\right) \subset C_{k} \subset B\left(0, \frac{(1+\varepsilon) k}{\mu}\right) \mid 0 \in C_{\infty}\right) \\
& \quad \geq 1-\exp (-c k),
\end{aligned}
$$

when $k$ is large enough. 
The shape theorem says that, for large $k, C_{k}$ looks like a big 'ball'. Corollary 3.1 below implies that, given a large enough constant $c>0$, with high probability, there is no large hole of radius $c(\log k)^{1 /(d-1)}$ in $C_{k}$. Specifically, given any $\varepsilon>0$, every big ball contained in $B(0,(1-\varepsilon) k / \mu)$ with radius larger than $c(\log k)^{1 /(d-1)}$ has points in $C_{k}$.

We make some necessary preparations and obtain the preliminary lemmas in Section 3 . The proofs of our main results are given in Section 4.

Throughout this paper, $c, c_{1}, c_{2}, \ldots$ denote positive finite constants that may change from line to line according to the context.

\section{Preliminary results}

First let us recall some basic results of percolation theory. We will use these results frequently in this paper. With the help of Theorem 3.1 below we can convert a problem concerning dependent percolation into a problem concerning Bernoulli bond percolation. We use Theorems 3.1 and 3.2 and Proposition 3.1, below, to obtain large deviation results for some variables in continuum percolation.

For $0 \leq \pi \leq 1$, let $Z^{\pi}=\left\{Z_{x}^{\pi}: x \in \mathbb{Z}^{d}\right\}$ be a family of independent random variables satisfying

$$
\mathrm{P}\left(Z_{x}^{\pi}=1\right)=1-\mathrm{P}\left(Z_{x}^{\pi}=0\right)=\pi \text { for all } x \in \mathbb{Z}^{d} .
$$

Theorem 3.1. (Theorem 7.65 of [7].) Let $d, k \geq 1$. There exists a nondecreasing function $\pi:[0,1] \rightarrow[0,1]$ satisfying $\pi(\delta) \rightarrow 1$ as $\delta \rightarrow 1$ such that the following assertion holds. If $Y=\left\{Y_{x}: x \in \mathbb{Z}^{d}\right\}$ is a $k$-dependent family of random variables satisfying

$$
\mathrm{P}\left(Y_{x}=1\right) \geq \delta \text { for all } x \in \mathbb{Z}^{d},
$$

then we have the stochastic domination

$$
Y \geq_{\mathrm{st}} Z^{\pi(\delta)}
$$

Let $B:=\prod_{i=1}^{d}\left[a_{i}, b_{i}\right]$ be a box in $\mathbb{R}^{d}$. We say that a component $C$ contained in $B$ is crossing for $B$ if, for all $i \in\{1,2, \ldots, d\}$, there exist vertices $x(i)=\left(x_{1}(i), x_{2}(i), \ldots, x_{d}(i)\right) \in C$ and $y(i)=\left(y_{1}(i), y_{2}(i), \ldots, y_{d}(i)\right) \in C$ such that $\left|x_{i}(i)-a_{i}\right| \leq \frac{1}{2}$ and $\left|y_{i}(i)-b_{i}\right| \leq \frac{1}{2}$. We often call $C$ a crossing component in $B$.

Proposition 3.1. (Proposition 10.13 of [14].) Suppose that $\lambda>\lambda_{\mathrm{c}}$, and that $\left(\phi_{s}, s \geq 0\right)$ is increasing with $\left(\phi_{s} / \log s\right) \rightarrow \infty$ as $s \rightarrow \infty$ and $\phi_{s} \leq s$ for all $s$. Let $E_{s}$ denote the event that

(i) there is a unique component of $G\left(\mathscr{H}_{\lambda, s} ; 1\right)$ that is crossing for $B(s)$, and

(ii) no other component of $G\left(\mathscr{H}_{\lambda, s} ; 1\right)$ has diameter greater than $\phi_{s}$.

Then $\lim \sup _{s \rightarrow \infty} \phi_{s}^{-1} \log \mathrm{P}\left(E_{s}^{\mathrm{c}}\right)<0$.

For any graph $G$, let $L_{j}(G)$ denote the order of its $j$ th-largest component; if it has fewer than $j$ components then $L_{j}(G)=0$.

Theorem 3.2. (Theorem 10.19 of [14].) Suppose that $\lambda>\lambda_{\mathrm{c}}$ and that $0<\varepsilon<\frac{1}{2}$. Let $E_{s}$ be the event that

(i) $L_{2}\left(G\left(\mathscr{H}_{\lambda, s} ; 1\right)\right)<\varepsilon \lambda p_{\infty}(\lambda) s^{d}$, and

(ii) $(1-\varepsilon) \lambda p_{\infty}(\lambda) \leq s^{-d} L_{1}\left(G\left(\mathscr{H}_{\lambda, s} ; 1\right)\right) \leq(1+\varepsilon) \lambda p_{\infty}(\lambda)$. 
Then there exist constants $c_{1}>0$ and $s_{0}>0$ such that

$$
\mathrm{P}\left(E_{s}^{\mathrm{c}}\right) \leq \exp \left(-c_{1} s^{d-1}\right), \quad s \geq s_{0} .
$$

Moreover, there is a lower bound of the form $\exp \left(-c_{2} s^{d-1}\right)$ (with $c_{2}>0$ ) for the probability that property (i) fails.

Theorem 3.3. (Theorem 10.20 of [14].) Suppose that $\lambda>\lambda_{c}, \varepsilon \in\left(0, \frac{1}{2}\right)$, and that $\left(\phi_{s}, s \geq 0\right)$ satisfies $\left(\phi_{s} / \log s\right) \rightarrow \infty$ as $s \rightarrow \infty$ and $\phi_{s} \leq s / 2$ for all $s$. Let $G_{s}$ denote the event that there exists a unique component $C_{b}(B(s))$ of $G\left(\mathscr{H}_{\lambda, s} ; 1\right)$ of metric diameter at least $\phi_{s}$. Let $E_{s}^{\prime}$ be the event that $G_{s}$ holds, and, additionally, let the order of $C_{b}(B(s))$ satisfy

$$
(1-\varepsilon) \lambda p_{\infty} \leq s^{-d}\left|C_{b}(B(s))\right| \leq(1+\varepsilon) \lambda p_{\infty} .
$$

Then there exist constants $c_{1}>0, c_{2}>0$, and $s_{0}>0$ such that

$$
\exp \left(-c_{1} \phi_{s}\right) \leq \mathrm{P}\left(G_{s}^{\mathrm{c}}\right) \leq \mathrm{P}\left(\left(E_{s}^{\prime}\right)^{\mathrm{c}}\right) \leq \exp \left(-c_{2} \phi_{s}\right), \quad s \geq s_{0} .
$$

Now we prove some lemmas and preliminary results which lead to our main results.

Lemma 3.1. Suppose that $\lambda>\lambda_{\mathrm{c}}$ and that $A$ is a Borel set in $\mathbb{R}^{d}$. Then there exists a constant $c>0$ such that, for all large $k$, we have

$$
\begin{aligned}
& \mathrm{P}\left(\text { there exists a component } C \subset G\left(\mathscr{H}_{\lambda} ; 1\right), k \leq|C|<\infty, C \cap A \neq \varnothing\right) \\
& \quad \leq \lambda|A| \exp \left(-c k^{(d-1) / d}\right) .
\end{aligned}
$$

Proof. Let $N(k)$ denote the number of vertices of $\mathscr{H}_{\lambda} \cap A$ lying in finite components of order at least $k$, and let $C_{x}$ be a component of $G\left(\mathscr{H}_{\lambda, x} ; 1\right)$ which contains $x$. Then

$$
\begin{aligned}
& \left.\mathrm{P} \text { (there exists a component } C \subset G\left(\mathscr{H}_{\lambda} ; 1\right), k \leq|C|<\infty, C \cap A \neq \varnothing\right) \\
& \quad \leq \mathrm{E}(N(k)) \quad \text { (using Palm theory-see Theorem } 1.6 \text { of [14]) } \\
& \quad=\int_{A} \lambda \mathrm{P}_{x}\left(k \leq\left|C_{x}\right|<\infty\right) \mathrm{d} x \\
& \quad=\lambda|A| \sum_{i=k}^{\infty} p_{i}(\lambda) .
\end{aligned}
$$

Using Theorem 10.15 of [14], we can obtain the correct bound of the above term and complete our proof.

Lemma 3.2. Suppose that $\lambda>\lambda_{\mathrm{c}}$. There exists a constant $c>0$ such that, for any $x \in \mathbb{R}^{d}$ with large $\|x\|$,

$$
\mathrm{P}_{0, x}\left(0 \leftrightarrow x, 0 \notin C_{\infty}\right) \leq \exp (-c\|x\|) .
$$

Proof. For any $x \in \mathbb{R}^{d}$ with large $\|x\|$, we have

$$
\mathrm{P}_{0, x}\left(0 \leftrightarrow x, 0 \notin C_{\infty}\right)
$$

$\leq \mathrm{P}$ (there exists a path $\ell$ from a point in $B(0,1)$ to a point in $\left.B(x, 1), \ell \not \subset C_{\infty}\right)$

$\leq \mathrm{P}($ there exists a component $C$ of metric diameter at least $\|x\|-2$ in $B(\|x\|)$,

$\left.C \not \subset C_{\infty}\right)$

$\leq \mathrm{P}($ there exists a component $C$ of metric diameter at least $\|x\|-2$ in $B(\|x\|)$,

$$
\left.|C| \leq \frac{1}{2} \lambda p_{\infty}(\lambda)\|x\|^{d}\right)
$$

$+\mathrm{P}\left(\right.$ there exists a component $C$ in $\left.B(\|x\|),|C|>\frac{1}{2} \lambda p_{\infty}(\lambda)\|x\|^{d}, C \not \subset C_{\infty}\right)$. 
Then, using Theorem 3.3 and Lemma 3.1, there exist constants $c_{1}>0$ and $c_{2}>0$ such that, for large $\|x\|$, we have

$$
\mathrm{P}_{0, x}\left(0 \leftrightarrow x, 0 \notin C_{\infty}\right) \leq \exp \left(-c_{1}\|x\|\right)+\exp \left(-c_{2}\|x\|^{d-1}\right) .
$$

Lemma 3.3. Suppose that $\lambda>\lambda_{\mathrm{c}}$. Then there exists a constant $c>0$ such that, for all large $r$,

$$
\mathrm{P}\left(B(0, r) \cap C_{\infty}=\varnothing\right) \leq \exp \left(-c r^{d-1}\right) .
$$

Proof. Clearly, $B(2 r / \sqrt{d}) \subseteq B(0, r)$. Let $C_{1}=C_{1}(B(2 r / \sqrt{d}))$ be the largest component of $G\left(\mathscr{H}_{\lambda, 2 r / \sqrt{d}} ; 1\right)$. Then we have

$$
\begin{aligned}
\mathrm{P}\left(B(0, r) \cap C_{\infty}=\varnothing\right) \leq & \mathrm{P}\left(B\left(\frac{2 r}{\sqrt{d}}\right) \cap C_{\infty}=\varnothing\right) \\
\leq & \mathrm{P}\left(\left|C_{1}\right|<\frac{1}{2}\left(\frac{2 r}{\sqrt{d}}\right)^{d} \lambda p_{\infty}(\lambda)\right) \\
& +\mathrm{P}\left(\left|C_{1}\right| \geq \frac{1}{2}\left(\frac{2 r}{\sqrt{d}}\right)^{d} \lambda p_{\infty}(\lambda), C_{1} \not \subset C_{\infty}\right) .
\end{aligned}
$$

Then, by Theorem 3.2 and Lemma 3.1, there exist constants $c_{1}>0$ and $c_{2}>0$ such that, for large $r$,

$$
\mathrm{P}\left(B(0, r) \cap C_{\infty}=\varnothing\right) \leq \exp \left(-c_{1} r^{d-1}\right)+\exp \left(-c_{2} r^{d-1}\right) .
$$

Corollary 3.1. Suppose that $\lambda>\lambda_{\mathrm{c}}$. Then there exists a constant $c>0$ such that, as $r \rightarrow \infty$,

$\mathrm{P}\left(\right.$ there exists $\left.B\left(x, c(\log r)^{1 /(d-1)}\right) \subset B(0, r), B\left(x, c(\log r)^{1 /(d-1)}\right) \cap C_{\infty}=\varnothing\right) \rightarrow 0$.

Proof. Clearly, there exists a constant $c_{1}>0$ such that, for all large enough constants $c>0$, there exists a collection of balls $\mathcal{B}:=\left\{B_{1, r}, B_{2, r}, \ldots, B_{m(r), r}\right\}$ of radius $c(\log r)^{1 /(d-1)} / 4 \mathrm{such}$ that, for all $r>0$,

$$
B(0, r) \subset \bigcup_{i=1}^{m(r)} B_{i, r},
$$

where $m(r)=c_{1} r^{d} /(\log r)^{d /(d-1)}$. Note that if a ball $B\left(x, c(\log r)^{1 /(d-1)}\right) \subset B(0, r)$, there certainly exists a ball $B_{i_{0}, r} \in \mathscr{B}$ such that $B_{i_{0}, r} \subset B\left(x, c(\log r)^{1 /(d-1)}\right)$. So we have

$$
\begin{aligned}
& \mathrm{P}\left(\text { there exists } B\left(x, c(\log r)^{1 /(d-1)}\right) \subset B(0, r), B\left(x, c(\log r)^{1 /(d-1)}\right) \cap C_{\infty}=\varnothing\right) \\
& \quad \leq \mathrm{P}\left(\bigcup_{i=1}^{m(r)}\left\{B_{i, r} \cap C_{\infty}=\varnothing\right\}\right) \\
& \quad \leq \sum_{i=1}^{m(r)} \mathrm{P}\left(B_{i, r} \cap C_{\infty}=\varnothing\right) .
\end{aligned}
$$

By Lemma 3.3, there exists a constant $c_{2}>0$ such that, for sufficiently large $r$,

$$
\sum_{i=1}^{m(r)} \mathrm{P}\left(B_{i, r} \cap C_{\infty}=\varnothing\right) \leq \frac{c_{1} r^{d}}{(\log r)^{d /(d-1)}} \exp \left(-c_{2} c^{d-1} \log r\right) .
$$

So if we choose a large enough constant $c>0$, the right-hand side of the above equation tends to 0 when $r$ tends to $\infty$. 
Lemma 3.4. Suppose that $\lambda>\lambda_{\mathrm{c}}$. Then there exists a constant $\rho=\rho(\lambda, d) \geq 1$ such that

$$
\limsup _{\|x\|_{1} \rightarrow \infty} \frac{\log \mathrm{P}_{0, x}\left(0 \leftrightarrow x, D(0, x) \geq \rho\|x\|_{1}\right)}{\|x\|_{1}}<0 .
$$

Furthermore, there exist constants $\rho_{1}>0$ and $c>0$ such that, for all $x \in \mathbb{R}^{d}$, all $t \geq \rho_{1}\|x\|$, and large enough $t$, we have

$$
\mathrm{P}_{0, x}(0 \leftrightarrow x, D(0, x) \geq t) \leq \exp (-c t)
$$

Proof. This lemma corresponds to Theorem 1.1 of [1], which is a similar result about the chemical distance in Bernoulli bond percolation. Equation (3.1) is a parallel result of Corollary 2.2 of [6]. Our proof mainly follows the strategy used in the proof of Theorem 1.1 of [1], but an additional argument is needed. Now we develop a renormalization technique for continuum percolation. First let us introduce some additional notation. Given a finite subset $\Lambda \in \mathbb{Z}^{d}$, define the outer boundaries of $\Lambda$ by

$$
\partial^{\text {out }} \Lambda:=\left\{x \in \Lambda^{\mathrm{c}}: \text { there exists a } y \in \Lambda,\{x, y\} \in \mathbb{E}^{d}\right\} .
$$

Given $M>0$, let the random field $\left(X_{z}, z \in \mathbb{Z}^{d}\right)$ be defined as follows. Define concentric cubes $B_{z}$ and $B_{z}^{+}$centered at the point $M z$ by $B_{z}:=B(M) \oplus\{M z\}$ and $B_{z}^{+}:=B\left(\frac{5}{4} M\right) \oplus\{M z\}$. If $\left\|z-z^{\prime}\right\|_{\infty}=1$, we write $z \sim^{*} z^{\prime}$. Recall the definition of the crossing component, which was defined immediately before Proposition 3.1. Define the event

$$
A_{z}:=\left\{\text { there exists a unique crossing component } C_{z} \text { in } B_{z}^{+}\right. \text {, }
$$
each subbox $B(M / 4) \oplus(z+h), h \in\{M / 2,-M / 2\}^{d}$, contains a unique crossing component, and all of these crossing components are connected to $\left.C_{z}\right\}$,

and set

$$
X_{z}:= \begin{cases}1 & \text { if } A_{z} \text { occurs } \\ 0 & \text { otherwise }\end{cases}
$$

It is obvious that $\left(X_{z}, z \in \mathbb{Z}^{d}\right)$ is a two-dimensional, dependent random field. By Proposition 3.1, the probability that $B_{z}^{+}$and each subbox $B(M / 4) \oplus(z+h) \subset B_{z}^{+}$contains a unique crossing component tends to 1 as $M \rightarrow \infty$. The probability that all the metric diameters of the components in $B_{z}^{+}$are smaller than $M / 10$ except $C_{z}$ tends to 1 as $M \rightarrow \infty$. So we obtain $\mathrm{P}\left(A_{z}\right) \rightarrow 1$ as $M \rightarrow \infty$. Given $0<\delta<1$, we can choose $M_{\delta}$ so that, as long as $M \geq M_{\delta}, \mathrm{P}\left(X_{z}=1\right) \geq \delta$ for all $z$. Therefore, by Theorem 3.1 we can choose $M_{0}>0$ such that, as long as $M \geq M_{0}$, we have the stochastic domination

$$
\left(X_{z}, z \in \mathbb{Z}^{d}\right) \geq_{\mathrm{st}}\left(Z_{z}^{p_{1}}, z \in \mathbb{Z}^{d}\right),
$$

where $\left(Z_{z}^{p_{1}}, z \in \mathbb{Z}^{d}\right)$ is a family of independent variables taking the value 1 with probability $p_{1}$ and 0 otherwise. We call the site $z \in \mathbb{Z}^{d}$ white if $X_{z}=1$, and black otherwise.

Now we introduce some definitions similar to those given in [1]. Consider two points $x, y \in \mathscr{H}_{\lambda}$. Let $a(x)$ and $a(y)$ be the almost-sure unique sites of the renormalized lattice such that $x \in B_{a(x)}$ and $y \in B_{a(y)}$. Set $n:=\|a(x)-a(y)\|_{1}$, and choose a macroscopic path $\mathfrak{A}:=a_{0} a_{1} \cdots a_{n}$ with $a_{0}=a(x)$ and $a_{n}=a(y)$. We denote by $\mathfrak{C}_{a}^{*}$ the $*$-connected macroscopic black cluster containing $a$, with $\mathfrak{C}_{a}^{*}=\varnothing$ if $a$ is white. We will use the convention that, for a 
white site $a$, we define $\partial^{\text {out }} \mathfrak{C}_{a}^{*}=\{a\}$. Define $\mathbb{C}:=\left\{\mathfrak{C}_{a}^{*}, a \in \mathfrak{A}\right\}$, which is the set of $*$-connected black clusters intersecting $\mathfrak{A}$. Define

$$
W:=\bigcup_{a \in \mathfrak{A}} \bigcup_{b \in \overline{\mathfrak{C}}_{a}^{-*}} B_{b}^{+},
$$

where $\overline{\mathfrak{C}}_{a}^{*}:=\mathfrak{C}_{a}^{*} \cup \partial^{\text {out }} \mathfrak{C}_{a}^{*}$. If $0 \leftrightarrow x$, let $y$ be the origin. We can use the method in [1] to construct a short path $\ell_{0}$ between 0 and $x$ in $W$. Since the path construction process of continuum percolation is exactly the same as Bernoulli bond percolation, we omit the details. Differing from the Bernoulli bond percolation, we have to do additional work with the path $\ell_{0}$. To ensure its length is small enough, we will make a slight modification. Suppose that two points $x_{1}$ and $x_{t}$ belong to a path $\ell$ in $B(s)$. Then there exists a path $\ell^{\prime}:=x_{1} x_{2} \cdots x_{t-1} x_{t} \subseteq \ell$ such that $B\left(x_{i}, \frac{1}{2}\right)$ overlaps only with $B\left(x_{i-1}, \frac{1}{2}\right), 1<i \leq t$, and $B\left(x_{i+1}, \frac{1}{2}\right), 1 \leq i<t$. Since $B(s+1)$ contains the $\lceil t / 2\rceil$ disjoint balls $B\left(x_{1}, \frac{1}{2}\right), B\left(x_{3}, \frac{1}{2}\right), \ldots$, we have $\frac{1}{2} t\left|B\left(0, \frac{1}{2}\right)\right| \leq|B(s+1)|$, from which we know that there exists a constant $c_{2}>0$ such that $t \leq c_{2}|B(s)|$ when $s$ is large enough. Therefore, by refining $\ell_{0}$ we can find a new path $\ell_{0}^{\prime} \subseteq \ell_{0} \in W$ connecting 0 and $x$, and a constant $c_{3}>0$ such that $\left|\ell_{0}^{\prime}\right| \leq \sum_{B_{z}^{+} \subseteq W} c_{2}\left|B_{z}^{+}\right| \leq|W| / c_{3}$. So we have

$$
\mathrm{P}_{0, x}\left(0 \leftrightarrow x, D(0, x)>\rho\|x\|_{1}\right) \leq \mathrm{P}\left(|W|>\rho c_{3}\|x\|_{1}\right) .
$$

It is easy to see that there exists a constant $c_{4}>0$ such that

$$
|W|<M^{d} c_{4}\left(n+1+\sum_{\mathfrak{C}^{*} \in \mathbb{C}}\left|\mathfrak{C}^{*}\right|\right) .
$$

So, using (3.2), we obtain

$$
\begin{aligned}
\mathrm{P}\left(|W|>\rho c_{3}\|x\|_{1}\right) & \leq \mathrm{P}\left(n+1+\sum_{\mathfrak{C}^{*} \in \mathbb{C}}\left|\mathfrak{C}^{*}\right|>\rho c_{3} c_{4}^{-1} M^{-d}\|x\|_{1}\right) \\
& \leq \mathrm{P}_{p_{1}}\left(n+1+\sum_{\mathfrak{C}^{*} \in \mathbb{C}}\left|\mathfrak{C}^{*}\right|>\rho c_{3} c_{4}^{-1} M^{-d}\|x\|_{1}\right) .
\end{aligned}
$$

By the last arguments of the proof of Theorem 1.1 of [1], we know that the right-hand side (RHS) of (3.3) satisfies the bound

$$
\operatorname{RHS} \text { of }(3.3) \leq \mathrm{P}_{p_{1}}\left(\sum_{i=0}^{n}\left(\left|\tilde{\mathfrak{C}}_{i}^{*}\right|+1\right)>\rho c_{3} c_{4}^{-1} M^{-d}\|x\|_{1}\right),
$$

where the $\left(\widetilde{\mathfrak{C}}_{i}^{*}\right)$ are independent, identically distributed random sets such that there exists an $h>0$ with $\mathrm{E}\left(\exp \left\{h\left(\left|\tilde{\mathfrak{C}}_{i}^{*}\right|+1\right)\right\}\right)<\infty$. Note that $n \leq\|x\|_{1}$ for large $M$, so if we choose $\rho$ appropriately large then there exists a constant $c_{5}>0$ such that

$$
\text { RHS of }(3.3) \leq \exp \left(-c_{5}\|x\|_{1}\right) \quad \text { for large }\|x\|_{1} \text {. }
$$

To see (3.1), just replace $\rho\|x\|_{1}$ with $t$ in the above inequalities.

Lemma 3.5. Suppose that $\lambda>\lambda_{\mathrm{c}}$ and that $x, y \in \mathbb{R}^{d}$. There exists a constant $\mu \geq 1$ such that, for each $\varepsilon>0$,

$$
\lim _{\|x-y\| \rightarrow \infty} \mathrm{P}_{x, y}\left(\left|\frac{D(x, y)}{\|x-y\|}-\mu\right|>\varepsilon \mid x, y \in C_{\infty}\right)=0 .
$$


Proof. First we introduce some definitions. Given $x \in \mathbb{R}^{d}$, define

$$
\tilde{x}:=\arg \inf _{y \in C_{\infty}}\|x-y\| .
$$

Assume that $x=M e_{1}$ and $s>12 M+24$, where $M$ is a strict positive constant and $e_{1}:=$ $(1,0, \ldots, 0)$. Define the three events

$$
\begin{aligned}
A_{1}:= & \left\{\tilde{x} \in B\left(x, \frac{1}{4} s\right), \tilde{y} \in B\left(y, \frac{1}{4} s\right)\right\}, \\
A_{2}:= & \left\{\text { there exists a unique component } C_{b}(B(s)) \text { of } G\left(\mathscr{H}_{\lambda, s} ; 1\right)\right. \text { of metric } \\
& \text { diameter at least } \left.\frac{1}{6} s, \frac{1}{2} s^{d} \lambda p_{\infty}(\lambda) \leq\left|C_{b}(B(s))\right| \leq \frac{3}{2} s^{d} \lambda p_{\infty}(\lambda)\right\}, \\
A_{3}:= & A_{2} \cap\left\{\tilde{x}, \tilde{y} \in C_{b}(B(s))\right\} .
\end{aligned}
$$

Then we have

$$
\mathrm{P}\left(A_{1} A_{3}\right) \geq \mathrm{P}\left(A_{2}\right)-\mathrm{P}\left(A_{1}^{\mathrm{c}}\right)-\mathrm{P}\left(A_{1} A_{2} A_{3}^{\mathrm{c}}\right) .
$$

The event $A_{1} A_{2} A_{3}^{\mathrm{c}}$ cannot occur as, otherwise, there exists a point $v$ in $B(x, s / 4)$ or $B(y, s / 4)$ such that $v \in C_{\infty}, v \notin C_{b}(B(s))$. Since the metric diameter of the component of $G\left(\mathscr{H}_{\lambda, s} ; 1\right)$ which contains $v$ is larger than $s / 2-s / 4-M-2>s / 6$, there are two components with metric diameters larger than $s / 6$, which cannot occur on the event $A_{2}$. So $A_{1} A_{3}^{\mathrm{c}} A_{2}=\varnothing$. Then we know that

$$
\mathrm{P}\left(A_{1} A_{3}\right) \geq \mathrm{P}\left(A_{2}\right)-\mathrm{P}\left(A_{1}^{\mathrm{c}}\right) .
$$

By Theorem 3.3 and Lemma 3.3, there exists a constant $c>0$ such that, for all large enough $s$,

$$
\mathrm{P}\left(A_{2}\right)-\mathrm{P}\left(A_{1}^{\mathrm{c}}\right) \geq 1-\exp \left(-c_{1} s\right)-2 \exp \left(-c_{2} s^{d-1}\right) \geq 1-\exp (-c s) .
$$

It is obvious that if event $A_{1} A_{3}$ occurs then $\tilde{x}, \tilde{y} \in C_{b}(B(s))$ and

$$
D(\tilde{x}, \tilde{y}) \leq\left|C_{b}(B(s))\right| \leq \frac{3}{2} s^{d} \lambda p_{\infty}(\lambda) .
$$

So we have, for all $s>s_{0}$,

$$
\mathrm{P}\left(D(\tilde{x}, \tilde{y})>\frac{3}{2} s^{d} \lambda p_{\infty}(\lambda)\right) \leq \mathrm{P}\left(\left(A_{1} A_{3}\right)^{\mathrm{c}}\right) \leq \exp (-c s) .
$$

Now we can give an upper bound of $\mathrm{E}(D(\tilde{x}, \tilde{y}))$ :

$$
\mathrm{E}(D(\tilde{x}, \tilde{y}))=\sum_{k=0}^{\infty} \mathrm{P}(D(\tilde{x}, \tilde{y})>k) \leq N+\sum_{k=N}^{\infty} \exp \left(-c k^{1 / d}\right)<\infty .
$$

Here $N$ is a sufficiently large constant. For all $x, y \in \mathbb{R}$, define $D_{x, y}:=D\left(\widetilde{x e_{1}}, \widetilde{y e_{1}}\right)$. For all $x, y, z \in \mathscr{H}_{\lambda}$, note that if $\left(x, p_{1}, \ldots, y\right)$ is a path from $x$ to $y$ and $\left(y, q_{1}, \ldots, z\right)$ is a path from $y$ to $z$, then $\left(x, p_{1}, \ldots, y, q_{1}, \ldots, z\right)$ is a path from $x$ to $z$. This immediately yields the triangle inequality,

$$
D_{0, n} \leq D_{0, m}+D_{m, n},
$$

where $m, n \in \mathbb{Z}^{+}$and $0 \leq m<n$. By the definition of $D_{m, n}$ and Proposition 2.6 of [13], we have

(i) $\left\{D_{n k,(n+1) k}, n \geq 1\right\}$ is a stationary and ergodic sequence for each $k$,

(ii) the distribution of $\left\{D_{m, m+k}, k \geq 1\right\}$ does not depend on $m$. 
By (3.4) we know that

$$
0<\mathrm{E}\left(D_{0,1}\right)<\infty .
$$

By (i), (ii), (3.5), and (3.6), applying the subadditive ergodic theorem [12], we conclude that there exists a constant $\mu \geq 0$ such that

$$
\lim _{n \rightarrow \infty} \frac{D_{0, n}}{n}=\mu \quad \text { almost surely. }
$$

By Lemma 3.3, for each $\varepsilon>0$, we have

$$
\mathrm{P}\left(D_{0, n} \geq(1-2 \varepsilon) n\right) \geq \mathrm{P}\left(B(0, \varepsilon n) \cap C_{\infty} \neq \varnothing, B\left(n e_{1}, \varepsilon n\right) \cap C_{\infty} \neq \varnothing\right) \rightarrow 1 .
$$

Combining (3.7) and (3.8), we have $\mu \geq 1$. By (3.4) and its proof process, we know that, for all $x \in \mathbb{R}$, there exists a constant $H>0$ such that $\mathrm{E}\left(D_{[x], x}\right)<H$. So, for each $\varepsilon>0$,

$$
\mathrm{P}\left(D_{[x], x}>\varepsilon x\right) \leq \frac{H}{\varepsilon x} \rightarrow 0 \quad \text { as } x \rightarrow \infty .
$$

Combing (3.7), (3.9), and the inequality $D_{0,[x]}-D_{[x], x} \leq D_{0, x} \leq D_{0,[x]}+D_{[x], x}$, we have

$$
\frac{D_{0, x}}{x} \rightarrow \mu \geq 1 \quad \text { in probability as }\|x\| \rightarrow \infty .
$$

By (3.10) and the isotropic property of continuum percolation, we know that, for all $x, y \in \mathbb{R}^{d}$,

$$
\frac{D(\tilde{x}, \tilde{y})}{\|x-y\|} \rightarrow \mu \quad \text { in probability as }\|x-y\| \rightarrow \infty .
$$

Now we define events

$$
\begin{aligned}
A_{4}:= & \left\{\text { there exists no finite component } C \text { of } G\left(\mathscr{H}_{\lambda} ; 1\right) \text { such that } C \cap B(x, 1) \neq \varnothing\right. \\
& \quad \text { and } C \cap B(y, 1) \neq \varnothing\}, \\
A_{5}:= & \quad \text { for all } q_{1}, q_{2} \in B(x, 1) \cap \mathcal{H}_{\lambda} \text { and } q_{1} \leftrightarrow q_{2}, \text { we have } D\left(q_{1}, q_{2}\right) \leq \sqrt{\|x-y\|}, \\
& \left.\quad \text { for all } q_{1}^{\prime}, q_{2}^{\prime} \in B(y, 1) \cap \mathscr{H}_{\lambda} \text { and } q_{1}^{\prime} \leftrightarrow q_{2}^{\prime}, \text { we have } D\left(q_{1}^{\prime}, q_{2}^{\prime}\right) \leq \sqrt{\|x-y\|}\right\} .
\end{aligned}
$$

By (3.1), for large $\|x-y\|$, there exists a constant $c_{1}>0$ such that

$$
\begin{aligned}
& \text { P(there exist } \left.q_{1}, q_{2} \in B(x, 1) \cap \mathscr{H}_{\lambda}, q_{1} \leftrightarrow q_{2}, D\left(q_{1}, q_{2}\right)>\sqrt{\|x-y\|}\right) \\
& \quad \leq \int_{B(0,1)} \int_{B(0,1)} \lambda^{2} \mathrm{P}_{q_{1}, q_{2}}\left(q_{1} \leftrightarrow q_{2}, D\left(q_{1}, q_{2}\right)>\sqrt{\|x-y\|}\right) \mathrm{d} q_{1} \mathrm{~d} q_{2} \\
& \quad \leq \exp \left(-c_{1} \sqrt{\|x-y\|}\right) .
\end{aligned}
$$

Using the above inequality and Lemma 3.1, we obtain

$$
\mathrm{P}\left(A_{4} A_{5}\right) \rightarrow 1 \quad \text { as }\|x-y\| \rightarrow \infty .
$$

Given any $\varepsilon>0$,

$$
\begin{aligned}
\mathrm{P}_{x, y} & \left(\left|\frac{D(x, y)}{\|x-y\|}-\mu\right| \leq \varepsilon \mid x, y \in C_{\infty}\right) \\
& \geq \mathrm{P}_{x, y}\left(\left|\frac{D(x, y)}{\|x-y\|}-\mu\right| \leq \varepsilon, A_{4} A_{5} \mid x, y \in C_{\infty}\right) .
\end{aligned}
$$


On event $A_{4} A_{5}$ and $x, y \in C_{\infty}$, let $\left(x, v_{1}, \ldots, v_{m}, y\right)$ be a path that realizes $D(x, y)\left(G\left(\mathscr{H}_{\lambda} \cup\right.\right.$ $\{x, y\} ; 1))$. Then $v_{1}, v_{2} \in C_{\infty}\left(G\left(\mathscr{H}_{\lambda} ; 1\right)\right)$. So we have

$$
\begin{aligned}
\mid D(x, & y)\left(G\left(\mathscr{H}_{\lambda} \cup\{x, y\} ; 1\right)\right)-D(\tilde{x}, \tilde{y})\left(G\left(\mathscr{H}_{\lambda} ; 1\right)\right) \mid \\
& \leq D\left(v_{1}, \tilde{x}\right)\left(G\left(\mathscr{H}_{\lambda} ; 1\right)\right)+D\left(v_{2}, \tilde{y}\right)\left(G\left(\mathscr{H}_{\lambda} ; 1\right)\right)+2 \\
& \leq 3 \sqrt{\|x-y\|} .
\end{aligned}
$$

Hence,

$$
\begin{array}{r}
\text { RHS of (3.13) } \geq \frac{1}{\mathrm{P}_{x, y}\left(x, y \in C_{\infty}\right)} \mathrm{P}\left(\left|\frac{D(\tilde{x}, \tilde{y})}{\|x-y\|}-\mu\right| \leq \varepsilon-\frac{3}{\sqrt{\|x-y\|}}, A_{4} A_{5},\right. \\
\left.B(x, 1) \cap C_{\infty} \neq \varnothing, B(y, 1) \cap C_{\infty} \neq \varnothing\right) .
\end{array}
$$

Then, by

$$
\begin{aligned}
\mathrm{P}_{x, y}\left(x, y \in C_{\infty}\right)-\mathrm{P}\left(B(x, 1) \cap C_{\infty} \neq \varnothing, B(y, 1) \cap C_{\infty} \neq \varnothing\right) & \leq \mathrm{P}\left(A_{4}^{\mathrm{c}}\right) \\
& \rightarrow 0 \text { as }\|x-y\| \rightarrow \infty,
\end{aligned}
$$

(3.11), and (3.12), we obtain

$$
\mathrm{P}_{x, y}\left(\left|\frac{D(x, y)}{\|x-y\|}-\mu\right| \leq \varepsilon \mid x, y \in C_{\infty}\right) \rightarrow 1 \quad \text { as }\|x-y\| \rightarrow \infty .
$$

This completes the proof.

Suppose that $\lambda>\lambda_{\mathrm{c}}$. For $x=\left(x_{1}, \ldots, x_{d}\right) \in \mathbb{R}^{d}$, suppose that $x \in C_{\infty}$. For $s>x_{1}$, define the passage time from $x$ to a hyperplane $H_{s}$ as

$$
b_{x, s}:=\inf \left\{D(x, y): y=\left(y_{1}, \ldots, y_{d}\right) \in C_{\infty}, y_{1}>s\right\},
$$

where $H_{s}$ is orthogonal to the first-coordinate axis at $(s, 0, \ldots, 0)$.

Lemma 3.6. Suppose that $\lambda>\lambda_{\mathrm{c}}, 0 \in C_{\infty}$, and that $\mu$ is the constant given in Lemma 3.5. Then we have

$$
\lim _{s \rightarrow \infty} \frac{b_{0, s}}{s}=\mu \text { in probability (by measure } \mathrm{P}_{0} \text { ). }
$$

Proof. For simplicity of exposition, in the proofs of Lemma 3.6 and Lemma 3.7, we restrict ourselves to the case in which $d=2$; the proofs of the results pose no serious difficulty when $d \geq 3$. For notational simplicity, define $R:=[-1,0] \times[-r, r]$. We first prove that there exist constants $c_{1}>0$ and $c_{2}>0$ such that, for all large $r$,

$$
\mathrm{P}\left(\text { there exist } x, y \in C_{\infty} \cap R \text { such that } D(x, y)>c_{1} r\right) \leq \exp \left(-c_{2} r\right) .
$$

Suppose that $\rho_{1}$ is the constant given in Lemma 3.4. Let $c_{1}=3 \rho_{1}$. Then we have

$$
\begin{aligned}
& \mathrm{P}\left(\text { there exist } x, y \in C_{\infty} \cap R, D(x, y)>3 \rho_{1} r\right) \\
& \quad \leq \int_{R} \int_{R} \lambda^{2} \mathrm{P}_{x, y}\left(x \leftrightarrow y, D(x, y)>3 \rho_{1} r\right) \mathrm{d} x \mathrm{~d} y .
\end{aligned}
$$

It is easy to see that, for large $r$ and $x, y \in R, 3 \rho_{1} r \geq \rho_{1}\|x-y\|$. So, by (3.1) and the above inequality, we obtain (3.14). 
Now we prove that there exists a constant $c_{3}>0$ such that, for all large $r>0$,

$$
\mathrm{P}\left(R \cap C_{\infty}=\varnothing\right) \leq \exp \left(-c_{3} r\right)
$$

Obviously, for large $r$,

$$
\begin{aligned}
\mathrm{P}(R \cap & \left.C_{\infty}=\varnothing\right) \\
\leq & \mathrm{P}(\text { there exists no crossing component for } B(2 r)) \\
& +\mathrm{P}\left(\text { there exists a crossing component } C \text { of } G\left(\mathscr{H}_{\lambda, 2 r} ; 1\right), C_{b} \cap C_{\infty}=\varnothing\right) .
\end{aligned}
$$

By Proposition 3.1, there exists a constant $c_{4}>0$ such that $(3.16) \leq \exp \left(-c_{4} r\right)$ when $r$ is large enough. Furthermore,

$$
\begin{gathered}
(3.17) \leq \mathrm{P}\left(\text { there exists a crossing component } C \text { of } G\left(\mathscr{H}_{\lambda, 2 r}\right),|C|<\frac{1}{2} \lambda p_{\infty}(\lambda) 4 r^{2}\right) \\
+\mathrm{P}\left(\text { there exists a component } C \subset G\left(\mathcal{H}_{\lambda} ; 1\right), \frac{1}{2} \lambda p_{\infty}(\lambda) 4 r^{2} \leq|C|<\infty,\right. \\
\quad C \cap B(2 r)=\varnothing) .
\end{gathered}
$$

By Proposition 3.1 and Lemma 3.1, we know that there exists a constant $c_{5}>0$ such that (3.17) $\leq \exp \left(-c_{5} r\right)$ when $r$ is large enough. So (3.15) follows.

For each $\varepsilon>0$, we now estimate the probability of $\left\{(1-\varepsilon) \mu s \leq b_{0, s} \leq(1+\varepsilon) \mu s\right\}$. Define $R_{i}:=[s, s+1] \times[i \eta s,(i+1) \eta s],-N \leq i \leq N-1$, where $\eta=\min \left\{\varepsilon / 2 c_{1}, \varepsilon /(2+\varepsilon)\right\}, c_{1}$ is a constant given by (3.14), and $N=\min \left\{n \in \mathbb{N}: \sqrt{(n \eta)^{2}+1} \geq(1+\varepsilon) \mu\right\}$.

For all $x \in[s, s+1] \times(-\infty,+\infty) \backslash[s, s+1] \times[-N \eta s, N \eta s]$, by the definition of $N$ we have $\|x\|>(1+\varepsilon) \mu s$. Since $\eta \leq \varepsilon /(2+\varepsilon)$, we have, for all $x \in R_{0},(1+\varepsilon / 2)\|x\| \leq$ $(1+\varepsilon / 2) \sqrt{(s+1)^{2}+(\eta s)^{2}}<(1+\varepsilon) s$ when $s$ is sufficiently large. So, if $s$ is large enough, we have

$$
\begin{aligned}
& \mathrm{P}_{0}\left((1-\varepsilon) \mu s \leq b_{0, s} \leq(1+\varepsilon) \mu s \mid 0 \in C_{\infty}\right) \\
& \geq \mathrm{P}_{0}\left(\bigcap _ { i = - N } ^ { N - 1 } \left\{R_{i} \cap C_{\infty} \neq \varnothing \text {, for all } x \in R_{i} \cap C_{\infty},\right.\right. \\
& \left.\left.\left(1-\frac{\varepsilon}{2}\right) \mu\|x\| \leq D(0, x) \leq\left(1+\frac{\varepsilon}{2}\right) \mu\|x\|\right\} \mid 0 \in C_{\infty}\right) \\
& \geq \mathrm{P}_{0}\left(\bigcap _ { i = - N } ^ { N - 1 } \left\{\text { there exists } v \in R_{i} \cap C_{\infty},\left(1-\frac{\varepsilon}{4}\right) \mu\|v\| \leq D(0, v) \leq\left(1+\frac{\varepsilon}{4}\right) \mu\|v\|,\right.\right. \\
& \text { for all } \left.\left.x \in R_{i}, x \leftrightarrow v, D(v, x) \leq \frac{\varepsilon s}{4}\right\} \mid 0 \in C_{\infty}\right) \\
& \geq 1-\sum_{i=-N}^{N-1}\left(\mathrm{P}_{0}\left(R_{i} \cap C_{\infty}=\varnothing \mid 0 \in C_{\infty}\right)\right. \\
& +\mathrm{P}_{0}\left(R_{i} \cap C_{\infty} \neq \varnothing,\right. \\
& \text { for all } \left.v \in R_{i} \cap C_{\infty},|D(0, v)-\mu\|v\||>\frac{1}{4} \varepsilon\|v\| \mid 0 \in C_{\infty}\right) \\
& \left.+\mathrm{P}_{0}\left(\text { there exist } x, v \in R_{i} \cap C_{\infty}, D(x, v)>\frac{\varepsilon s}{4} \mid 0 \in C_{\infty}\right)\right) \text {. }
\end{aligned}
$$


Since the second term in the sum on the right-hand side of (3.18) is less than or equal to

$$
\sup _{v \in R_{i}}\left\{\mathrm{P}_{0, v}\left(v \in C_{\infty},|D(0, v)-\mu\|v\||>\frac{1}{4} \varepsilon\|v\| \mid 0 \in C_{\infty}\right)\right\}
$$

and $\varepsilon s / 4>c_{1} \eta s / 2$, by (3.15), Lemma 3.5, and (3.14), there exist constants $c_{6}>0$ and $c_{7}>0$ such that

$$
\begin{aligned}
& \mathrm{P}_{0}\left((1-\varepsilon) \mu s \leq b_{0, s} \leq(1+\varepsilon) \mu s \mid 0 \in C_{\infty}\right) \\
& \quad \geq 1-2 N\left(\exp \left(-c_{6} \eta s\right)+o(1)+\exp \left(-c_{7} \eta s\right)\right) \\
& \quad \rightarrow 1 \text { as } s \rightarrow \infty .
\end{aligned}
$$

Lemma 3.7. Suppose that $\lambda>\lambda_{\mathrm{c}}$ and that $\mu$ is the constant given in Lemma 3.5. For $s_{1}>0$ and $s_{2}=O\left(s_{1}\right)$, define the passage time by

$$
\begin{gathered}
l_{s_{1}, s_{2}}:=\inf \left\{D(x, y): x \in[-1,0] \times\left[-\frac{1}{2} s_{2}, \frac{1}{2} s_{2}\right]^{d-1} \cap \mathscr{H}_{\lambda},\right. \\
\left.y \in\left[s_{1}, s_{1}+1\right] \times(-\infty,+\infty)^{d-1} \cap \mathscr{H}_{\lambda}\right\} .
\end{gathered}
$$

Then we have

$$
\lim _{s_{1} \rightarrow \infty} \frac{l_{s_{1}, s_{2}}}{s_{1}}=\mu \quad \text { in probability. }
$$

Proof. As mentioned in the proof of Lemma 3.6, we focus on the case in which $d=2$. Define $R:=[-1,0] \times\left[-s_{2} / 2, s_{2} / 2\right]$. Since $s_{2}=O\left(s_{1}\right)$, there exists a constant $c_{3}>0$ such that $s_{2} / s_{1} \leq c_{3}$. Recall the definition of $b_{x, s}$, which was defined above Lemma 3.6. For each $\varepsilon>0$, we have

$$
\begin{aligned}
\mathrm{P}\left((1-\varepsilon) \mu s_{1} \leq l_{s_{1}, s_{2}} \leq(1+\varepsilon) \mu s_{1}\right) & \\
\geq & \mathrm{P}\left(R \cap C_{\infty} \neq \varnothing, \text { for all } x \in R \cap C_{\infty},\left|b_{x, s_{1}}-\mu s_{1}\right| \leq \varepsilon s_{1}\right) \\
& \quad-\mathrm{P}\left(\text { there exists a component } C \text { of } G\left(\mathscr{H}_{\lambda} ; 1\right), C \cap R \neq \varnothing, s_{1} \leq|C|<\infty\right) .
\end{aligned}
$$

Now we bound the two terms on the right-hand side of the above inequality. Let $N:=\left\lceil c_{1} c_{3} / \varepsilon\right\rceil$, where $c_{1}$ is a positive constant given by (3.14). Define $R_{i}:=[-1,0] \times\left[-s_{2} / 2+(i-\right.$ 1) $\left.s_{2} / N,-s_{2} / 2+i s_{2} / N\right], 1 \leq i \leq N$. For the first term,

$$
\begin{gathered}
\mathrm{P}\left(R \cap C_{\infty} \neq \varnothing, \text { for all } x \in R \cap C_{\infty},\left|b_{x, s_{1}}-\mu s_{1}\right| \leq \varepsilon s_{1}\right) \\
\geq \mathrm{P}\left(\bigcap_{i=1}^{N}\left\{R_{i} \cap C_{\infty} \neq \varnothing, \text { for all } x \in R_{i} \cap C_{\infty},\left|b_{x, s_{1}}-\mu s_{1}\right| \leq \varepsilon s_{1}\right\}\right) \\
\geq \mathrm{P}\left(\bigcap _ { i = 1 } ^ { N } \left\{\text { there exists } v \in R_{i} \cap C_{\infty},\left|b_{v, s_{1}}-\mu s_{1}\right| \leq \frac{\varepsilon s_{1}}{2}, \text { for all } x \in R_{i} \cap C_{\infty},\right.\right. \\
\left.\left.\quad D(v, x) \leq \frac{\varepsilon s_{1}}{2}\right\}\right) \\
\quad+\mathrm{P}\left(R_{i} \cap C_{\infty} \neq \varnothing, \text { for all } v \in R_{i} \cap C_{\infty},\left|b_{v, s_{1}}-\mu s_{1}\right|>\frac{\varepsilon s_{1}}{2}\right) \\
\left.\quad+\mathrm{P}\left(\text { there exists } x, y \in R_{i} \cap C_{\infty}, D(x, y)>\frac{\varepsilon s_{1}}{2}\right)\right) .
\end{gathered}
$$


Since the second term in the sum on the right-hand side of (3.19) is less than or equal to

$$
\sup _{v \in R_{i}}\left\{\mathrm{P}_{v}\left(v \in C_{\infty},\left|b_{v, s_{1}}-\mu s_{1}\right|>\frac{1}{2} \varepsilon s_{1}\right)\right\}
$$

and $\varepsilon s_{1}>c_{1} s_{2} / N$, by (3.15), Lemma 3.6, and (3.14), we know there exist constants $c_{4}>0$ and $c_{5}>0$ such that

$$
\begin{aligned}
\mathrm{P}(R & \left.\cap C_{\infty} \neq \varnothing, \text { for all } x \in R \cap C_{\infty},\left|b_{x, s_{1}}-\mu s_{1}\right| \leq \varepsilon s_{1}\right) \\
& \geq 1-N\left(\exp \left(-\frac{c_{4} s_{1}}{N}\right)+o(1)+\exp \left(-\frac{c_{5} s_{1}}{N}\right)\right) \\
& \rightarrow 1 \text { as } s_{1} \rightarrow \infty .
\end{aligned}
$$

For the second term, by Lemma 3.1 we have

$\mathrm{P}\left(\right.$ there exists a component $C$ of $\left.G\left(\mathscr{H}_{\lambda} ; 1\right), C \cap R \neq \varnothing, s_{1} \leq|C|<\infty\right) \rightarrow 0 \quad$ as $s_{1} \rightarrow \infty$.

\section{Final proofs}

Proof of Theorem 2.1. The proof is divided into two parts: the upper large deviations and the lower large deviations. First we prove that there exists a constant $c>0$ satisfying the upper large deviation inequality

$$
\mathrm{P}_{0, x}(0 \leftrightarrow x, D(0, x)>(1+\varepsilon) \mu\|x\|) \leq \exp (-c\|x\|),
$$

when $\|x\|$ is large enough. The lines of our main proof follow those in [5]. We now present some definitions and notation analogous to those in [5]. For all $x \in \mathbb{R}^{d}$, define the following set around $x$ :

$$
\mathcal{I}_{x}^{M}:=\left\{y \in B(x, \sqrt{M}) \cap \mathscr{H}_{\lambda}: y \leftrightarrow \partial B(x, M)\right\} .
$$

Here $y \leftrightarrow \partial B(x, M)$ means that there exists a path in $G\left(\mathscr{H}_{\lambda} ; 1\right)$ connecting $y$ and a point outside $\partial B(x, M)$. We define the related random variable $I_{x}^{M}$ by

$$
I_{x}^{M}:= \begin{cases}x & \text { if } \mathcal{I}_{x}^{M}=\varnothing, \\ \arg \inf _{y \in \mathcal{I}_{x}^{M}}\|x-y\| & \text { otherwise. }\end{cases}
$$

Recall the definition of $\tilde{x}$, which was defined in the proof of Lemma 3.5. By Lemma 3.1 and Lemma 3.3, we have

$$
\begin{aligned}
\mathrm{P}\left(I_{x}^{M} \neq \tilde{x}\right) \leq \mathrm{P}\left(\text { there exists a component } C \subset G\left(\mathcal{H}_{\lambda} ; 1\right), M-\sqrt{M} \leq|C|<\infty,\right. & \\
& C \cap B(x, \sqrt{M}) \neq \varnothing)+\mathrm{P}\left(B(x, \sqrt{M}) \cap C_{\infty}=\varnothing\right) \\
\rightarrow 0 & \text { as } M \rightarrow \infty .
\end{aligned}
$$

For all $x, y \in \mathbb{R}^{d}$, if $x$ or $y$ is not a Poisson point, let $D(x, y)=\infty$. So by (3.11) we obtain

$$
\frac{D\left(I_{x}^{\|x-y\|}, I_{y}^{\|x-y\|}\right)}{\|x-y\|} \rightarrow \frac{D(\tilde{x}, \tilde{y})}{\|x-y\|} \rightarrow \mu \text { in probability. }
$$


Now we introduce a dependent bond percolation $\left(X_{e}, e \in \mathbb{E}^{d}\right)$. For each edge $e=(x, y) \in \mathbb{E}^{d}$ and $\eta(\varepsilon)>0$, we define the event $R_{e}:=\left\{D\left(I_{M x}^{M}, I_{M y}^{M}\right) \leq M \mu(1+\eta)\right\}$ and set

$$
X_{e}:= \begin{cases}1 & \text { if } R_{e} \text { occurs } \\ 0 & \text { otherwise }\end{cases}
$$

It is easy to see that the states of the edges are finitely dependent. By (4.1) and Theorem 3.1, for each constant $p_{1}<1$, we can choose $M_{0}>0$ such that, as long as $M>M_{0}$, we have the stochastic domination

$$
\left(X_{e}, e \in \mathbb{E}^{d}\right) \geq_{\mathrm{st}}\left(Z_{e}^{p_{1}}, e \in \mathbb{E}^{d}\right),
$$

where $\left(Z_{e}^{p_{1}}, e \in \mathbb{E}^{d}\right)$ is an independent Bernoulli bond percolation. For each $z \in \mathbb{Z}^{d}$, each $x \in \mathbb{R}^{d}$, and each $r>0$, we respectively define the annuluses in $\mathbb{Z}^{d}$ and $\mathbb{R}^{d}$ as

$$
\begin{aligned}
& \mathcal{A}_{\mathbb{Z}^{d}}(z, r):=\left\{y \in \mathbb{Z}^{d}: \frac{1}{2} r \leq\|y-z\|_{1} \leq r\right\}, \\
& \mathcal{A}_{\mathbb{R}^{d}}(x, r):=\left\{y \in \mathbb{R}^{d}: \frac{1}{2} r \leq\|y-x\|_{1} \leq r\right\} .
\end{aligned}
$$

Suppose that $\rho$ is the constant given in Lemma 3.4. For each $\alpha=\eta / 2 \rho$, define an event $G$ in $\left(X_{e}, e \in \mathbb{E}^{d}\right)$ by

$$
G:=\left\{\text { there exist } a \in \mathcal{A}_{\mathbb{Z}^{d}}\left(0, \alpha\|z\|_{1}\right) \text { and } b \in \mathcal{A}_{\mathbb{Z}^{d}}\left(z, \alpha\|z\|_{1}\right), D(a, b) \leq(1+3 \alpha)\|z\|_{1}\right\},
$$

where $D(\cdot, \cdot)$ is the chemical distance defined in [5]. By (4.2) and Step 3 in the proof of Theorem 1.3 of [5], we know that, for large $M$, there exists a constant $c_{1}>0$ such that

$$
\mathrm{P}\left(G^{\mathrm{c}}\right) \leq \exp \left(-c_{1}\|z\|_{1}\right)
$$

when $\|z\|_{1}$ is large enough. The details of the proof are given in [5], and thus we omit them. Now suppose that $x \in \mathbb{R}^{d}$ is on the axis and that $\|x\|$ is sufficiently large. Then we can choose an appropriate $M$ such that $\|x\|$ is an integer multiple of $M$, i.e. $z=x / M$, and (4.3) holds. Suppose that the event $G$ occurs. Then, by the relationship between $\left(X_{e}, e \in \mathbb{E}^{d}\right)$ and $G\left(\mathscr{H}_{\lambda} ; 1\right)$, it is easy to show that there exists a path $\ell$ from some point $S \in D_{1}=\mathcal{A}_{\mathbb{R}^{d}}(0, \alpha\|x\|) \oplus B(0, \sqrt{M})$ to some point $T \in D_{2}=\mathcal{A}_{\mathbb{R}^{d}}(x, \alpha\|x\|) \oplus B(0, \sqrt{M})$ and that $|\ell| \leq(1+3 \alpha)(1+\eta) \mu\|x\|$. Given $\varepsilon>0$, we can choose the corresponding $\eta(\varepsilon)$ such that $2 \eta+(1+3 \alpha)(1+\eta) \mu \leq(1+\varepsilon) \mu$, which implies that

$$
\begin{aligned}
& G \cap\left\{S \in C_{\infty}\right\} \cap\{0 \leftrightarrow S, D(0, S) \leq \eta\|x\|\} \cap\{T \leftrightarrow x, D(T, x) \leq \eta\|x\|\} \\
& \subseteq\{0 \leftrightarrow x, D(0, x) \leq(1+\varepsilon) \mu\|x\|\} .
\end{aligned}
$$

Therefore,

$$
\begin{aligned}
\mathrm{P}_{0, x}(0 & \leftrightarrow x, D(0, x)>(1+\varepsilon) \mu\|x\|) \\
\leq & \left.\mathrm{P}_{0} \text { (there exists } S \in D_{1}, 0 \leftrightarrow S, D(0, S)>\eta\|x\|\right) \\
& \left.+\mathrm{P}_{x} \text { (there exists } T \in D_{2}, T \leftrightarrow x, D(T, x)>\eta\|x\|\right) \\
& +\mathrm{P}\left(\text { there exist } S \in D_{1}, T \in D_{2}, S \leftrightarrow T, S \notin C_{\infty}\right)+\mathrm{P}\left(G^{\mathrm{c}}\right) \\
\leq & \int_{D_{1}} \lambda \mathrm{P}_{0, S}(0 \leftrightarrow S, D(0, S)>\eta\|x\|) \mathrm{d} S+\int_{D_{2}} \lambda \mathrm{P}_{x, T}(T \leftrightarrow x, D(T, x)>\eta\|x\|) \mathrm{d} T \\
& +\int_{D_{1}} \int_{D_{2}} \lambda^{2} \mathrm{P}_{S, T}\left(S \leftrightarrow T, S \notin C_{\infty}\right) \mathrm{d} S \mathrm{~d} T+\mathrm{P}\left(G^{\mathrm{c}}\right) .
\end{aligned}
$$


Now we bound the above four terms. Since $S \in \mathcal{A}_{\mathbb{R}^{d}}(0, \alpha\|x\|) \oplus B(0, \sqrt{M})$ and $\alpha=\eta / 2 \rho$, by Lemma 3.4, there exists a constant $c_{2}>0$ such that

$$
\begin{gathered}
\mathrm{P}_{0, S}(0 \leftrightarrow S, D(0, S)>\eta\|x\|) \leq \mathrm{P}_{0, S}(0 \leftrightarrow S, D(0, S)>\rho\|S\|) \leq \exp \left(-c_{2}\|x\|\right), \\
\mathrm{P}_{x, T}(T \leftrightarrow x, D(T, x)>\eta\|x\|) \leq \exp \left(-c_{2}\|x\|\right),
\end{gathered}
$$

when $\|x\|$ is sufficiently large. By Lemma 3.13 we know that there exists a constant $c_{3}>0$ such that, for large $\|x\|$,

$$
\mathrm{P}_{S, T}\left(S \leftrightarrow T, S \notin C_{\infty}\right) \leq \exp \left(-c_{3}\|x\|\right) .
$$

Combining (4.3), (4.4), (4.5), and (4.6), we complete the proof of the upper large deviation inequality.

In the following, we prove that there exists a constant $c>0$ such that

$$
\mathrm{P}_{0, x}(0 \leftrightarrow x, D(0, x)<(1-\varepsilon) \mu\|x\|) \leq \exp (-c\|x\|),
$$

when $\|x\|$ is large enough. Garet and Marchand [5] used the method provided in the proof of Theorem 3.1 of [8] to obtain the lower large deviations results. For our continuum model, it is natural to expect that method would work also. It does. Similarly to the proof of the lower large deviations in [5], we just need to prove the lemmas, given in this paper as Lemmas 3.5-3.7, that appear in [8]. Garet and Marchand [5] considered all directions in $\mathbb{Z}^{d}$ for the Bernoulli bond percolation model, but here we just need to consider the case along the coordinate axes because of the isotropic property of the continuum percolation. Now we give some definitions analogous to those in [8]. For each $k=\left(k_{1}, k_{2}\right) \in \mathbb{Z}^{2}$ and integers $M$ and $N$ satisfying $M>N>1$, define

$$
\begin{aligned}
& S(k):=\left\{v \in \mathbb{R}^{2}: N k \leq v<N(k+1)\right\}, \\
& T(k):=\left\{v \in \mathbb{R}^{2}: N k-M \leq v<N(k+1)+M\right\} .
\end{aligned}
$$

Here $T(k)$ is a very large box and contains $S(k)$ at its center. Let $\ell:=(v(0), \ldots, v(v))$ be a path from $v(0)=0$ to a point $v(v) \in \mathscr{H}_{\lambda, 0} \cap\left\{x \in \mathbb{R}^{2}, x_{1}>n\right\}$. Then we can find the following two sequences related to $\ell$. Let $k(0)=0$ and $a(0)=0$, let $v(a(1))$ be the first vertex along $\ell$ with the property that $v(a) \notin T(k(0))$, and let $k(1)$ be the unique $k$ such that $v(a(1)) \in S(k)$. Continuing recursively we can obtain sequences $a(0), \ldots, a(\tau(\ell))$ and $k(0), \ldots, k(\tau(\ell))$ such that

1. $0=a(0)<a(1)<\cdots<a(\tau(\ell))<\nu$,

2. $v(a(i)) \in S(k(i))$,

3. $a(i+1)$ is the smallest integer $a$ larger than $a(i)$ such that $v(a) \notin T(k(i))$.

The final terms satisfy, for all $a(\tau(\ell)) \leq j \leq v$,

$$
v(j) \in T(k(\tau(\ell))) .
$$

By the process of 'loop removal' [8], we can remove the double points in $\sigma=(k(0), \ldots$, $k(\tau(\ell)))$ and obtain a subsequence of $\sigma$ :

$$
\tilde{\sigma}=(l(0), \ldots, l(\rho(\ell))),
$$


where $l(a)=k\left(j_{a}\right)$ and $0=j_{0}<j_{1}<\cdots<j_{\rho(\ell)} \leq \tau(\ell)$. Note that, although we may have $j_{\rho(\ell)}<\tau(\ell)$, it is always true that $k\left(j_{\rho(\ell)}\right)=k(\tau(\ell))$. Now consider the portion $\ell(i)=(v(a(i-1)), \ldots, v(a(i)))$ of $\ell$ which stretches between $S(k(i-1))$ and $S(k(i))$. Define

$$
L(i)=\max _{m=1,2}\left|v_{m}(a(i))-v_{m}(a(i-1))\right| .
$$

By construction, $M \leq L(i) \leq M+N$ for $1 \leq i \leq \tau$. For $1 \leq i \leq \rho(\ell)$, consider the portion $\ell\left(j_{i}\right)$ between $S\left(k\left(j_{i-1}\right)\right)$ and $S\left(k\left(j_{i}\right)\right)$, and color the vector $l(i)=k\left(j_{i}\right)$ white if

$$
\left|\ell\left(j_{i}\right)\right| \leq(\mu-2 \varepsilon) L\left(j_{i}\right)
$$

and black otherwise. Let $\omega$ be the white points in the sequence $(l(1), \ldots, l(\rho(\ell)))$.

Now we prove the lemmas that correspond to those used in the proof of Theorem 3.1 of [8]. The proofs of the lemmas given in this paper as Lemmas 3.5 and 3.6 are exactly the same, and so we just need to prove the lemma given in this paper as Lemma 3.7.

Suppose that $0<\varepsilon<\mu / 5$. Define $p=p(M, N, \varepsilon)$ to be the probability that some point $x \in C_{\infty} \cap S(0)$ is joined to some point $y$ outside the square $[-M+N, M)^{2}$, and let $D(x, y)<(M+N)(\mu-2 \varepsilon)$. Then we should have

$$
p(M, N, \varepsilon) \rightarrow 0 \quad \text { as } M, N \rightarrow \infty,
$$

so long as $M \geq N(2 \mu / \varepsilon)$. Note that our notation $l_{m, n}$ corresponds to the notation $l_{n m}$ in [8]. Following the lines of the proof in [8] we have

$$
p(M, N, \varepsilon) \leq 4 \mathrm{P}\left(l_{M-N, 3(M-N)} \leq(M-N)(\mu-\varepsilon)\right) .
$$

Therefore, by Lemma 3.7 we have $p(M, N, \varepsilon) \rightarrow 0$ as $M-N \rightarrow \infty$. So the corresponding lemmas in [8] are all proved and the proof is thus complete.

Proof of Theorem 2.2. The proof follows naturally from the uniform estimates in Theorem 2.1. First, it is easy to see that

$$
\begin{aligned}
1-\mathrm{P}_{0} & \left(\left(C_{\infty} \cap B\left(0, \frac{(1-\varepsilon) k}{\mu}\right)\right) \subset C_{k} \subset B\left(0, \frac{(1+\varepsilon) k}{\mu}\right) \mid 0 \in C_{\infty}\right) \\
\leq & \mathrm{P}_{0}\left(C_{k} \not \subset B\left(0, \frac{(1+\varepsilon) k}{\mu}\right) \mid 0 \in C_{\infty}\right) \\
& +\mathrm{P}_{0}\left(C_{k} \not \supset\left(C_{\infty} \cap B\left(0, \frac{(1-\varepsilon) k}{\mu}\right)\right) \mid 0 \in C_{\infty}\right) .
\end{aligned}
$$

Let us now estimate each term separately. By Palm theory,

$$
\begin{aligned}
\mathrm{P}_{0}( & \left.C_{k} \not \subset B\left(0, \frac{(1+\varepsilon) k}{\mu}\right) \mid 0 \in C_{\infty}\right) \\
& =\mathrm{P}_{0}\left(\text { there exists } x \in C_{\infty},\|x\|>\frac{(1+\varepsilon) k}{\mu}, D(0, x) \leq k \mid 0 \in C_{\infty}\right) \\
& \leq \int_{\|x\|>(1+\varepsilon) k / \mu} \lambda \mathrm{P}_{0, x}\left(0 \leftrightarrow x, D(0, x) \leq k \mid 0 \in C_{\infty}\right) \mathrm{d} x .
\end{aligned}
$$


So, using Theorem 2.1, there exists a constant $c_{1}>0$ such that

$$
\mathrm{P}_{0}\left(C_{k} \not \subset B\left(0, \frac{(1+\varepsilon) k}{\mu}\right) \mid 0 \in C_{\infty}\right)<\exp \left(-c_{1} k\right),
$$

when $k$ is large enough.

Now we estimate the second term. Suppose that $\rho_{1}$ is the constant given in Lemma 3.4. Let $\rho_{2}>\rho_{1}$ and $1 / \rho_{2}<(1-\varepsilon) / \mu$. Then we have

$$
\begin{aligned}
\mathrm{P}_{0}\left(C_{k}\right. & \left.\not \supset\left(C_{\infty} \cap B\left(0, \frac{(1-\varepsilon) k}{\mu}\right)\right) \mid 0 \in C_{\infty}\right) \\
= & \mathrm{P}_{0}\left(\text { there exists } x \in C_{\infty},\|x\| \leq \frac{(1-\varepsilon) k}{\mu}, D(0, x)>k \mid 0 \in C_{\infty}\right) \\
\leq & \int_{\|x\| \leq k / \rho_{2}} \lambda \mathrm{P}_{0, x}\left(0 \leftrightarrow x, D(0, x)>k \mid 0 \in C_{\infty}\right) \mathrm{d} x \\
& +\int_{k / \rho_{2}<\|x\| \leq(1-\varepsilon) k / \mu} \lambda \mathrm{P}_{0, x}(0 \leftrightarrow x, D(0, x)>k) \mathrm{d} x .
\end{aligned}
$$

By the above inequality, using (3.1) and Theorem 2.1, we can choose a constant $c_{2}>0$ such that, for large $k$,

$$
\mathrm{P}_{0}\left(C_{k} \not \supset\left(C_{\infty} \cap B\left(0, \frac{(1-\varepsilon) k}{\mu}\right)\right) \mid 0 \in C_{\infty}\right) \leq \exp \left(-c_{2} k\right) .
$$

Now we have given the correct bounds to the two terms, completing the proof.

\section{Acknowledgements}

The authors thank the anonymous referee for valuable comments and remarks. This research was supported by the National Natural Science Foundation of China (grant number 10831006) and the Knowledge Innovation Program of the Chinese Academy of Sciences (grant number kjcx-yw-s7).

\section{References}

[1] Antal, P. And Pisztora, A. (1996). On the chemical distance for supercritical Bernoulli percolation. Ann. Prob. 24, 1036-1048.

[2] Baccelli, F. and Bordenave, C. (2007). The radial spanning tree of a poisson point process. Ann. Appl. Prob. 17, 305-359.

[3] Dousse, O., Mannersalo, P. and Thiran, P. (2004). Latency of wireless sensor networks with uncoordinated power saving mechanisms. In Proc. 5th ACM Internat. Symp. Mobile Adhoc Networking Computing (MOBIHOC '04), Association for Computing Machinery, New York, pp. 109-120.

[4] Garet, O. and Marchand, R. (2004). Asymptotic shape for the chemical distance and first-passage percolation on the infinite Bernoulli cluster. ESAIM Prob. Statist. 8, 169-199.

[5] Garet, O. And Marchand, R. (2007). Large deviations for the chemical distance in supercritical Bernoulli percolation. Ann. Prob. 35, 833-866.

[6] Garet, O. And Marchand, R. (2010). Moderate deviations for the chemical distance in Bernoulli percolation. ALEA Latin Amer. J. Prob. Math. Statist. 7, 171-191.

[7] Grimmetr, G. (1999). Percolation, 2nd edn. Springer, Berlin.

[8] Grimmett, G. And Kesten, H. (1984). First-passage percolation, network flows and electrical resistances. Z. Wahrscheinlichkeitsth. 66, 335-366.

[9] Hammersley, J. M. AND Welsh, D. J. A. (1965). First-passage percolation, subadditive processes, stochastic networks, and generalized renewal theory. In Proc. Internat. Res. Semin. (Statist. Lab., University of California, Berkeley), Springer, New York, pp. 61-110. 
[10] Howard, C. D. And Newman, C. M. (1997). Euclidean models of first-passage percolation. Prob. Theory Relat. Fields 108, 153-170.

[11] Howard, C. D. and Newman, C. M. (2001). Geodesics and spanning trees for Euclidean first-passage percolation. Ann. Prob. 29, 577-623.

[12] Liggett, T. M. (1985). An improved subadditive ergodic theorem. Ann. Prob. 13, 1279-1285.

[13] Meester, R. and Roy, R. (1996). Continuum Percolation. Cambridge University Press.

[14] Penrose, M. (2003). Random Geometric Graphs. Oxford University Press.

[15] Stoyan, D., Kendall, W. S. And Mecke, J. (1995). Stochastic Geometry and Its Applications, 2nd edn. John Wiley, Chichester.

[16] Vahidi-Asl, M. Q. And Wierman, J. C. (1992). A shape result for first-passage percolation on the Voronoi tessellation and Delaunay triangulation. In Random Graphs, Vol. 2, John Wiley, New York, pp. 247-262. 